\title{
Automatic Parallelization of Fine-Grained Metafunctions on a Chip Multiprocessor
}

\author{
SANGHOON LEE and JAMES TUCK, North Carolina State University
}

Due to the importance of reliability and security, prior studies have proposed inlining metafunctions into applications for detecting bugs and security vulnerabilities. However, because these software techniques add frequent, fine-grained instrumentation to programs, they often incur large runtime overheads. In this work, we consider an automatic thread extraction technique for removing these fine-grained checks from a main application and scheduling them on helper threads. In this way, we can leverage the resources available on a CMP to reduce the latency and overhead of fine-grained checking codes.

Our parallelization strategy extracts metafunctions from a single threaded application and executes them in customized helper threads-threads constructed to mirror relevant fragments of the main program's behavior in order to keep communication and overhead low. To get good performance, we consider optimizations that reduce communication and balance work among many threads.

We evaluate our parallelization strategy on Mudflap, a pointer-use checking tool in GCC. To show the benefits of our technique, we compare it to a manually parallelized version of Mudflap. We run our experiments on an architectural simulator with support for fast queueing operations. On a subset of SPECint 2000, our automatically parallelized code using static load balance is only $19 \%$ slower, on average, than the manually parallelized version on a simulated eight-core system. In addition, our automatically parallelized code using dynamic load balance is competitive, on average, to the manually parallelized version on a simulated eightcore system. Furthermore, all the applications except parser achieve better speedups with our automatic algorithms than with the manual approach. Also, our approach introduces very little overhead in the main program-it is kept under $100 \%$, which is more than a $5.3 \times$ reduction compared to serial Mudflap.

Categories and Subject Descriptors: D.3.4 [Programming Languages]: Processors-Compilers, code generation; C.1.4 [Computer System Organization]: Processor Architectures-Parallel architectures; D.1.3 [Programming Techniques (E)]: Concurrent Programming-Parallel programming

General Terms: Design, Algorithms, Experimentation, Performance

Additional Key Words and Phrases: Automatic parallelization, helper computing, chip multiprocessor

\section{ACM Reference Format:}

Lee, S. and Tuck, J. 2013. Automatic parallelization of fine-grained metafunctions on a chip multiprocessor. ACM Trans. Architec. Code Optim. 10, 4, Article 30 (December 2013), 26 pages.

DOI: http://dx.doi.org/10.1145/2541228.2541237

\section{INTRODUCTION}

One major trend in computing is the continuing increase in the complexity of software systems. Such an increase is motivated by the expectation of increasingly powerful hardware (faster processors, larger memory, etc.), programming environments that provide higher abstraction to the programmers, and the increasing diversity of

This work is supported in part by the National Science Foundation, under grant CNS-0834664.

Authors' addresses: S. Lee (now at Qualcomm, in Morrisville, NC) and J. Tuck, Department of Electrical and Computer Engineering, North Carolina State University, Raleigh, NC 27695-7256; email: \{shlee5, jtuck\}@ ncsu.edu.

Permission to make digital or hard copies of part or all of this work for personal or classroom use is granted without fee provided that copies are not made or distributed for profit or commercial advantage and that copies show this notice on the first page or initial screen of a display along with the full citation. Copyrights for components of this work owned by others than ACM must be honored. Abstracting with credit is permitted. To copy otherwise, to republish, to post on servers, to redistribute to lists, or to use any component of this work in other works requires prior specific perimssion and/or a fee. Permissions may be requested from Publications Dept., ACM, Inc., 2 Penn Plaza, Suite 701, New York, NY 10121-0701, USA, fax: +1 (212) 869-0481 or permission@acm.org.

(c) 2013 ACM 1544-3566/2013/12-ART30 $\$ 15.00$

DOI: http://dx.doi.org/10.1145/2541228.2541237 
environments in which the software runs. Programmers increasingly rely on metafunctions, functionality that does not contribute directly to the output of a program but helps ensure efficient, reliable, and secure execution of the program. Various forms of metafunctions are implemented today in response to the diverse requirements of software execution. In all, metafunctions are very diverse and include garbage collection, security checks, runtime correctness verification, application-level checkpointing, performance instrumentation, and many more.

At the same time, the trend in microprocessor design is toward many-core systems. The performance of a single core in a many-core system will increase at a slower speed than in the past, and in some cases will stagnate. In order to exploit the performance of such a system, programmers need to exploit parallelism at various levels in their applications.

Software's increasing reliance on metafunctions presents a promising source of parallelism. The predominate approach in performing metafunctions is to interleave their execution with the main application code, through direct augmentation of the metafunction code into the application code or by multiplexing the processor at runtime between the application and its metafunction execution. Such an interleaved execution approach invariably increases the critical path of the main application, sometimes increasing it by orders of magnitudes. For example, Memcheck, a tool that runs using Valgrind, has been reported to slow down applications by a factor of $2 \times$ to $30 \times$ [Newsome and Song 2005; Valgrind Developers 2005; Seward and Nethercote 2005], and even then it only checks for a subset of memory errors.

Parallelism has been exploited for performing metafunctions, such as memory management [Kharbutli et al. 2006; Tiwari et al. 2010] and bug detection [Shetty et al. 2006; Nightingale et al. 2008]. However, these techniques mainly focused on long-running, coarse-grained metafunctions.

In this work, we focus on automatically extracting metafunctions from singlethreaded programs and then scheduling them for parallel execution on multicores. We presume the compiler knows a priori which metafunctions can execute in parallel with the main program, but it must extract the relevant control and data dependences to execute the metafunction in parallel. Furthermore, it must balance the execution of multiple metafunctions across multiple threads to achieve low latency and scalability on a multicore processor.

One of the primary challenges for fine-grained metafunctions is the abundant instructions inserted throughout the main application. Reducing this fine-grained overhead is difficult to accomplish while still providing scalability. Our strategy is the generation of customized helpers that follow the calling context of the main program and replicate as much state as possible in a way that does not require communication. Using a smart task partitioning strategy, the metafunction code and some of its dependences are migrated to the customized helper. This approach results in low overhead in the main thread since metafunctions and their support code need not be executed there. To provide scalability, we generate multiple customized helpers using static and dynamic load-balancing heuristics.

In our work, we make the following contributions:

-We propose a novel parallelization methodology using Custom Helpers for finegrained metafunction parallelization.

- To provide scalability, we describe static and dynamic heuristics to generate multiple helpers and balance their load.

-We evaluate our framework on Mudflap, a pointer-use checking tool implemented in GCC. We run our experiments on an architectural simulator modeling an aggressive eight-core CMP with support for fast queueing operations. We show that our automatic technique is efficient and scalable. With static heuristics, it achieves 
an average $1.64 \times$ speedup using eight cores. Compared to a manually optimized version of the Mudflap framework, our approach is only $19 \%$ slower. With dynamic heuristics, it achieves an average $1.96 \times$ speedup using eight cores. Our automatically parallelized code using dynamic load balance is competitive, on average, to the manually parallelized version on a simulated eight-core system. Furthermore, our custom helpers reduce the overhead in the main thread by $5.3 \times$ compared to serial Mudflap.

The remainder of the article is organized as follows: Section 2 describes the key ideas behind our compiler and parallelization strategy. Section 3 presents our compiler algorithms. Section 4 discusses requirements of the metafunction library. Section 5 explains how Mudflap is mapped into our framework. The experimental setup and evaluation are in Section 6. Related work is given in Section 7. Section 8 briefly discusses some limitations of our work. Section 9 concludes.

\section{FINE-GRAINED METAFUNCTION PARALLELIZATION}

Metafunctions are extra functionality added to a program to augment its behavior in some useful way. While they can be an arbitrary function, we restrict their definition, somewhat, to cover common uses and to limit the scope of our study. We assume that metafunctions have the following properties: (1) They are placed at a program point, $p$, to compute some desired property at that location. (2) They take as input State $p$ that is extracted from the main program state and MetaState $p_{i n}$ that is information calculated specifically by the metafunctions. A critical simplifying assumption is that MetaState is disjoint from State. (3) They produce as output an optional error state, Error $_{p}$, and optional updates to the metafunction state, MetaState $p_{\text {out }}$. Note that none of these metafunctions update State; we do not allow metafunctions to update program state in order to narrow the scope of this work.

We will assume that most metafunctions fall into two categories: validating metafunctions and updating metafunctions. A validating metafunction checks a property

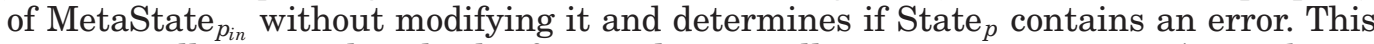
is essentially a complex check of some dynamically invariant property. An updating metafunction uses State $_{p}$ to transition MetaState $p_{p_{i n}}$ to MetaState pout $_{\text {ou }}$ and may detect an error related to the update operation. For example, in pointer-use checking, an important validating metafunction is testing that a dereference occurs to memory that has been allocated and not freed; an example of an updating metafunction is tracking when an object is allocated or freed so that the database of known memory objects is kept up-to-date.

There are several costs associated with inserting metafunctions into a program. First and foremost, the metafunction must be executed as part of the main program, thereby adding considerable runtime overhead. This instruction payload can increase runtime by orders of magnitude. Second, the insertion of code and function calls worsens the compiler's ability to optimize the main program. As a result, the efficiency of the main application is much worse than it would be without metafunction instrumentation. The first two effects are clearly discernible from direct inspection of the generated code. Third, the additional code competes with the main program code for critical processor resources, like issue slots, space in the cache memory hierarchy, or space in branch predictor history tables.

Table I shows some key overheads from Mudflap, a pointer-use checking tool. All percentages are shown relative to a binary without any Mudflap support. This data was collected using the same experimental setup described in Section 6 . The fraction of updating and validating metafunctions combined is approximately $42 \%$ (Check + Other); this fraction represents time spent in the computational region of 
Table I. Characterization of Mudflap Overheads Normalized to an Optimized Baseline Without It

\begin{tabular}{|c|c|c|c|c|c|c|}
\hline \multirow[b]{2}{*}{ Application } & \multirow{2}{*}{$\begin{array}{c}\text { Norm } \\
\text { Execution } \\
\text { (Cycles) }\end{array}$} & \multirow{2}{*}{$\begin{array}{c}\text { Norm } \\
\text { Dynamic } \\
\text { Inst. Count }\end{array}$} & \multicolumn{3}{|c|}{$\%$ of Dynamic Instruction Count } & \multirow{2}{*}{$\begin{array}{c}\text { Check } \\
\text { Average Inst } \\
\text { Count }\end{array}$} \\
\hline & & & Check & Other & Hash & \\
\hline bzip2 & 11.1 & 19.2 & 51.8 & 14.2 & 25.3 & 1988.2 \\
\hline gap & 6.6 & 9.7 & 21.1 & 5.4 & 53.6 & 1646.0 \\
\hline gzip & 3.9 & 6.2 & 52.0 & 0.0 & 26.7 & 2011.6 \\
\hline mcf & 5.6 & 15.7 & 55.1 & 0.0 & 34.1 & 1945.4 \\
\hline parser & 7.1 & 11.0 & 15.9 & 22.3 & 39.7 & 1871.3 \\
\hline twolf & 6.1 & 10.0 & 12.7 & 12.3 & 53.3 & 1664.5 \\
\hline vpr & 5.4 & 8.2 & 25.6 & 22.7 & 33.1 & 1994.7 \\
\hline Geo.Mean. & 6.2 & 10.7 & 28.7 & 13.7 & 36.5 & 1868.7 \\
\hline
\end{tabular}

metafunctions. Additional inlined code makes up another 36\% (Hash) of the program. The total increase in instructions, on average, is $10.7 \times$.

A naive way to parallelize metafunction workloads is by rewriting the metafunctions to explicitly leverage parallelism. Such a metafunction-level approach to parallelization is straightforward to achieve. In such a parallelization strategy, each metafunction would decide the best way to parallelize its task. For example, it may send all of its inputs to waiting helper threads that are ready to receive and process them. At a minimum, it will need to send State ${ }_{p}$. With frequent metafunction operations, the main thread will spend considerable time forwarding tasks to helpers. This approach can achieve good performance, but it requires significant programmer effort and cannot exploit redundancy or eliminate inefficiency that may exist across metafunctions within the same program.

\subsection{Our Parallelization Approach: Custom Helpers}

In our approach, we want to extract metafunction code out of the main program and run it in parallel. By automatically extracting metafunction code to helper threads, we can construct highly tuned, custom helpers that match the main program's needs. Looking at each input to a metafunction provides some insight on how to do this.

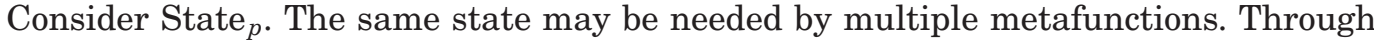
code analysis, the compiler can generate a custom helper, which receives the input value once and uses it in each metafunction call. Furthermore, rather than communicating just the inputs to metafunctions, the main can communicate values for a code slice that the helper uses to generate the full State ${ }_{p}$ for one or more metafunction calls. By sending only a few inputs for a slice, some work in the main thread can be eliminated and communication with the helper can be reduced. Location ${ }_{p}$ can also be handled more efficiently. If a custom helper is generated for a specific region of code, then some inputs can be embedded directly in the helper without any need for communication.

In summary, our compiler will generate a custom helper thread that is highly aware of the main program. It would be possible to generate a custom helper for the entire program (essentially a reduced program clone) or just for parts of it. So that we do not need to choose regions for parallelization, we generate a custom helper for the entire main program. The helper will mimic the behavior of the main program by matching its call stack dynamically. Relationships among multiple metafunctions will be exploited through a task selection algorithm that aggressively moves or replicates code from the main thread into the custom helper.

\subsection{Providing Scalability}

Given the large computational overhead introduced through metafunctions, multiple threads are needed to successfully parallelize them. For example, as shown in 
Table I, there is no hope of significantly reducing the overhead in bzip2 with only one helper thread. Ideally, performance should scale as additional resources are added; once enough resources are added, the impact on the main program should be minimal. Therefore, we support multiple custom helpers.

In order to support multiple threads, metafunction parallelism must be exposed to the compiler. A general way of expressing and operating on such parallelism is beyond the scope of this article. Currently, we make decisions about which metafunctions can run in parallel when the compiler is compiled (i.e., it is hard-coded) to narrow the scope of the article. In other words, they are fixed for a given set of metafunctions. To decide which metafunctions can run in parallel, we leveraged profiling information and our understanding of the metafunction implementation as much as possible. ${ }^{1}$ The metafunction requirements are discussed more in Section 4.

To provide scalability, multiple custom helpers can be generated for the same function. Each custom thread will receive part of the metafunction workload of a function. In order to use multiple helpers as efficiently as possible, load balance must be considered. Therefore, we provide simple static algorithms and show that they work reasonably well. The two heuristics we consider are random assignment and round robin. However, load balance is challenging with only a static algorithm for a variety of reasons. First, metafunctions may have dynamically varying execution times. For example, in order to reduce the time of metafunction execution, memoizaton is often used to significantly reduce overheads. As a result, the same metafunction can have wildly varying execution times. Second, the execution path of the program is unknown and can only be estimated. To properly balance load, you want the metafunctions that will actually execute to be distributed evenly. Finally, the structure of some program regions may not lend themselves to parallelization over many threads. For example, an inner loop may have one metafunction. If the loop is not unrolled and the trip count is high, then all executions of the inner loop body will be scheduled on the same helper, resulting in imbalance. Analogous behaviors occur with many different code structures.

To compensate for all of these effects due to a static algorithm, we provide dynamic algorithms to balance workloads more evenly and show that the dynamic algorithms work better than the static algorithms. Our dynamic algorithms are based on broadcasting. We describe the detailed algorithms in Section 3.5.

\subsection{Summary}

In summary, our overall parallelization approach aims to reduce the overhead in the main application by extracting customized helpers that provide low overhead metafunction execution. However, to combat the high overheads of metafunctions themselves, we leverage multiple helpers to scale up to many threads and keep the overhead in the main program low. We will show that this approach is competitive with manual parallelization in our case study.

\section{PARALLELIZATION ALGORITHM}

We have designed a compiler algorithm that extracts and schedules metafunctions to run in parallel. In the explanation that follows, we start with the parallelization of a single function that includes metafunctions. Then, we expand it to support generation of the base helper thread, which mimics the main thread's behavior. Next, we describe how to use the base helper thread parallelization algorithm for custom helper thread extraction. Finally, we describe how to scale the algorithm to multiple threads using static and dynamic load balancing techniques.

\footnotetext{
${ }^{1}$ This is nontrivial to perform automatically, but the implementer of such metafunctions can determine parallelization properties with reasonable effort.
} 


\subsection{Helper Function Parallelization Algorithm}

We base our parallelization algorithm for a single function on the Program Dependence Graph (PDG) [Ferrante et al. 1987]. We include all control, register, and memory dependence edges in our PDG. The CreateHelper algorithm in Algorithm 1 shows the algorithm for creating the helper function.

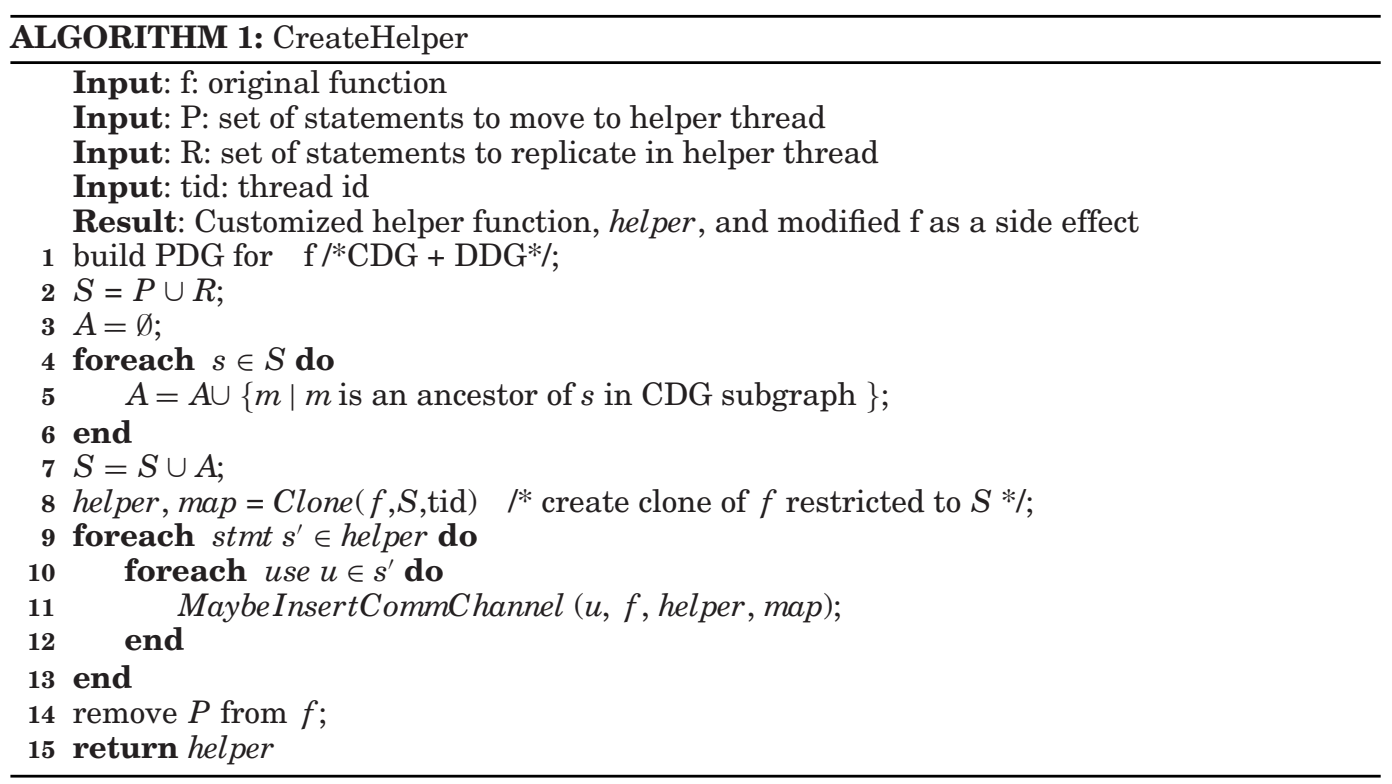

The inputs to Create Helper are the original function in SSA form $(f)$, a subset of statements $P$ to include only in the helper, and a subset of statements $R$ to include in both. We assume that extracting $P$ and $R$ to the helper will not result in cyclic communication between the main program and the helper thread; rather, communication flows in a single direction from the main thread to the helper. CreateHelper assumes that $P$ and $R$ have been selected properly, so no such check is repeated here.

In order to move a statement to the helper, a few actions are required. First, we build a PDG [Ferrante et al. 1987] (a unified control and data dependence graph) for $f$. We set $S=P \cup R$ so that we have all statements to be included in the helper in one place. Next, all control predicates for each statement in $S$ must be included in the helper (lines 4-7). Once all the nodes in the helper are computed, a clone of the function can be created with only the selected statements and control predicates present (line 8). For each input or predicate value, a communication channel, in the form of a queue, is added between the function and the helper (lines 9-12). In line 14, instructions in $P$ are removed from $f$.

MaybeInsertCommChannel has some smarts built into it that are not shown here. First, we check if $u$ really needs to be communicated: is def $f(u)$ in $f$ but not in helper? This test covers three cases. Either the definition has been moved to helper, it has been replicated in helper, or it is only in $f$. We only communicate $u$ if it is not available in helper. If we do need to communicate $u$, we proceed as follows. Since $f$ is in SSA form, we insert a send $(u)$ just after its definition in $f$, and we replace its definition in helper with $u=\operatorname{rec} v()$. We also keep a record that $u$ was communicated and never insert another channel for it between $f$ and helper.

One additional detail worth noting. Clone forces the new helper function to have a signature with no input parameters and no return value. This is not strictly necessary, 
but it did simplify the implementation of the compiler since all helper functions have the same type of signature and do not need arguments (which would complicate Algorithm 4 presented in the next section).

\subsection{Baseline Helper Extraction}

Our baseline helper is a clone of the entire main program that mimics the main application's call stack. This ensures the helper is very lean and efficient by default. Whenever the main application calls a function, the helper should call and execute an associated helper version.

The algorithm is shown in several parts. First, in the Base HelperThread function (Algorithm 2), we accumulate all call statements into the $R$ set for parallelization. Next, we generate the helper with those statements extracted using the CreateHelper algorithm. CreateHelper works as previously described, but we can now consider more of the details in the Clone function, shown in Algorithm 3. Clone will copy the function exactly; however, it will remove all statements that are not in $S$, and it will replace all call statements that are not metafunctions with a code sequence that will call their associated helper.
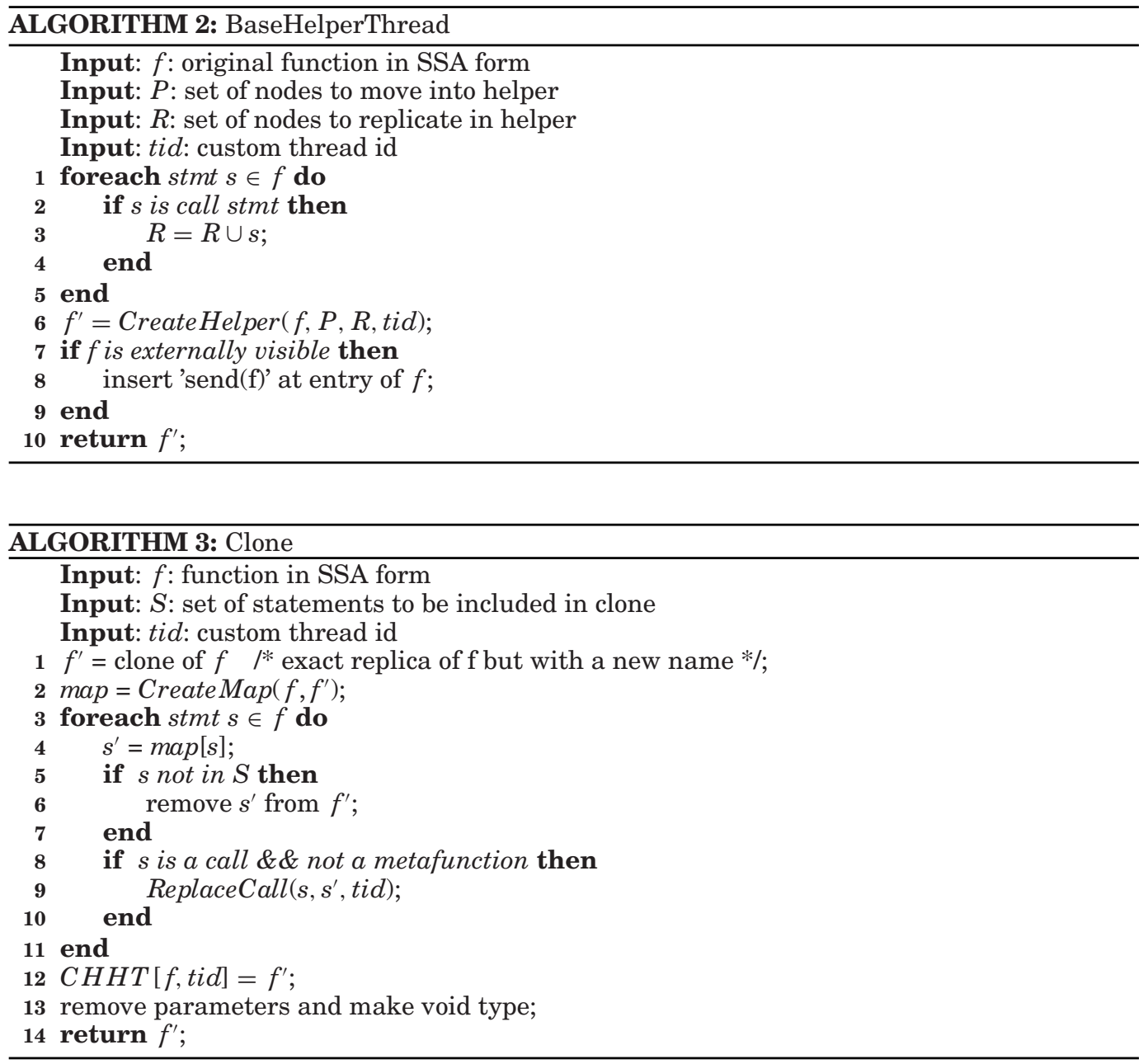
Replacing the calls turns out to be more involved than it may appear at first. There are several cases that must be supported for this to work for arbitrary programs. First, indirect function calls through pointers can only be resolved at runtime. Therefore, the mechanism for determining which helper to run must occur at runtime (at least some of the time). Second, functions in libraries may not have a corresponding helper. This happens because the library's code was unavailable to the compiler, and it could not generate a cloned version of it. Ideally, in these cases, this can be prevented by providing a wrapper function for the library call that makes it compatible with our system, but this is not always possible. So, the external library call will not have a corresponding helper, but it could still call yet another function that does have an associated helper. The qsort function is a good example; a function pointer is passed as an argument and is used to help sort the data structure. Such a function passed as an argument is now externally visible. The qsort function may not have a helper, but its function argument likely will. While neither case is very common, both do occur with regularity in applications and must be supported.

Custom Helper Hash Table. In order to dynamically bind a function with its customized helper, a Custom Helper Hash Table (CHHT) is constructed, which creates a mapping between a function and its helper. ${ }^{2}$ Since we may generate multiple custom helper threads, it also needs a thread id as input.

The CHHTLookup function shown in Algorithm 4 is used at runtime to find the dynamic mapping of function to its custom helper. There are several cases that are required for correctness. We will consider each case in turn.

Direct and Indirect Function Calls. To support direct and indirect calls, at each call site we must determine the mapping from the callee to the helper thread. We can accomplish this with the CHHTLookup function by passing the address of the callee as an argument. In the case of the indirect call, the computation of the callee's address must be sent to the helper as a result of lines 9 through 12 in CreateHelper. For direct calls, the callee's address can be encoded directly in the helper. The relevant lines of code are 5 and 6 in Algorithm 4, and 9 in Algorithm 5.

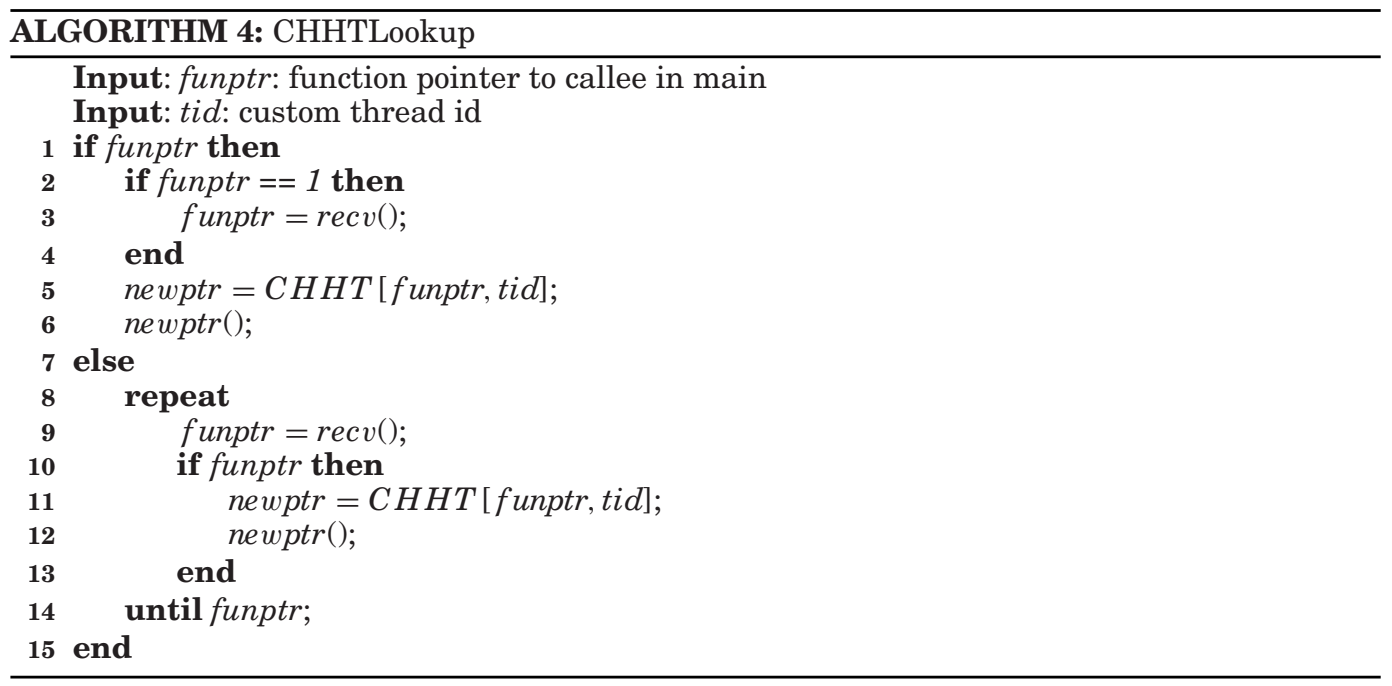

${ }^{2}$ To create the $C H H T$, the compiler builds a sequence of code to be executed at the beginning of the program that initializes the $C H H T$ and inserts all known mappings. 


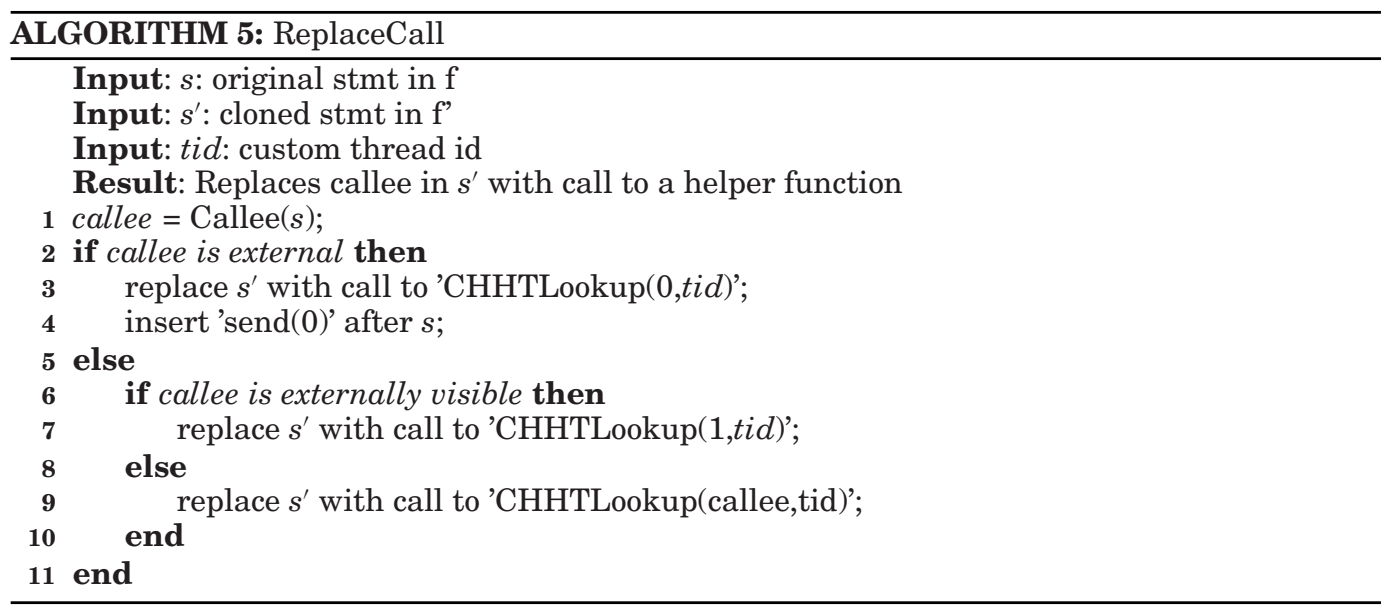

While these mechanisms work well in C programs where indirect function calls are rare, they may perform poorly in languages or programs where such calls are common. For example, object-oriented programs that make heavy use of virtual functions will suffer from this additional overhead.

Externally Visible Functions. Since we cannot modify all of the call sites of externally visible functions, we must communicate with the helper from inside the function rather than at the call site. At such a call site that we can analyze, we replace the call with CHHTLookup with a value of 1 as funptr (6-7 in Algorithm 5). This will force CHHTLookup to wait until it receives one function pointer (line 2) from the called function; then it uses it to perform the lookup and call the corresponding helper function.

When BaseHelperThread analyzes the externally visible function, it inserts the 'send(f)' code (lines 7-9 in Algorithm 2).

External Calls. If an externally defined function (like sort) is called and there is no CHHT entry or wrapper function available, then the helpers have no work to do and will be idle during its execution. However, the external function may call an externally visible function that does have a helper. In this case, while the main executes the external call, the helper calls CHHTLookup with a 0 as its funptr argument. This will force the helper to wait until it receives a function pointer from an externally visible function (lines 7-14 in Algorithm 4). However, because the external function may never call such a function or it may call such functions repeatedly, we must identify when CHHTLookup terminates by sending a null pointer after the external call ends (lines 2-4 in Algorithm 5).

Optimized Call Sequence. This mechanism works for all function calls, but it is sometimes inefficient. With additional information about the function, these costs can be avoided. For example, in the case of a static function in $\mathrm{C}$, we need not call CHHTLookup at runtime to determine the mapping. Instead, we can hard-code the helper function's location directly into its caller and allow function inlining to further optimize the code. We do not show it in our algorithm, but we do take this additional step in our full implementation.

\subsection{Custom Helper Extraction}

In Custom Helper Extraction, metafunctions and their support code are extracted from a function in the main thread and scheduled in the customized helper thread. We assume that a function, $f$, is already annotated with all metafunctions and supporting 


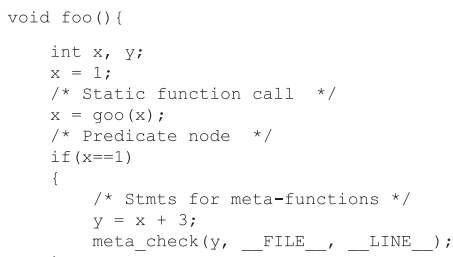

(a) Original version

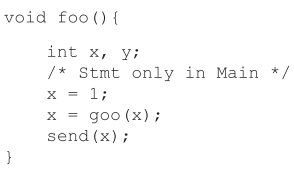

(b) Main thread version

(c) Helper thread version (tid=1)

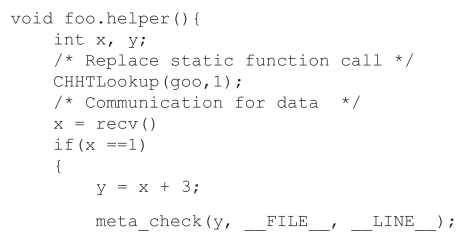

Fig. 1. A simple example of our Helper Extraction.

code, and we assume that metafunction calls can be identified and inserted into a set, $M$, of statement nodes. Using only $M$ as the basis for helper extraction would yield an inefficient extraction with all metafunction inputs communicated explicitly to the helper. Instead, we take a smarter approach and look for additional statements to include in $M$ that will reduce the communication overhead between $f$ and the helper.

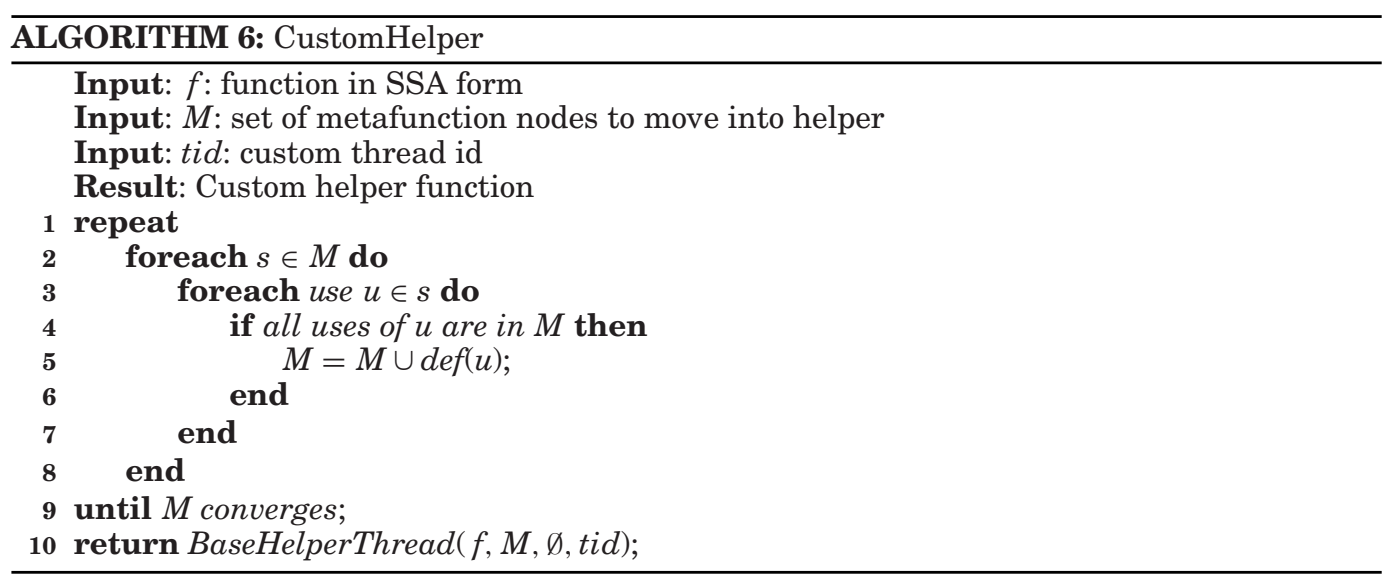

Our algorithm is shown in Algorithm 6. Using SSA form, we efficiently iterate over every statement $s$ in $M$. For each use $u$ in $s$; if $u$ is only used by statements in $M$, then the definition of $u$ can be added to $M$ as long as it adds no extra communication (or even reduces it). The extraction phase finishes once all members of $M$, both new ones and ones added along the way, have been considered. This algorithm will see to it that any data only computed for the metafunction is included in $M$. Finally, we use the CreateHelper function to parallelize the statements in $M$ by setting the input argument $P=M$. Note, this algorithm will never create cyclic communication between $f$ and the helper.

Interestingly, a valuable benefit of this technique is the extraction of context-sensitive constant values. For example, many metafunctions take code location as an argument. For a given custom helper, code location amounts to a constant value that will easily be extracted by the algorithm since it is only used in the metafunction.

\subsection{Example}

Figure 1 shows a simple example of our Helper Extraction. Figure 1(a) shows the original function. Using our algorithms, we generate functions for the main thread (Figure 1(b)) and the helper thread (Figure 1(c)), respectively. This example illustrates 


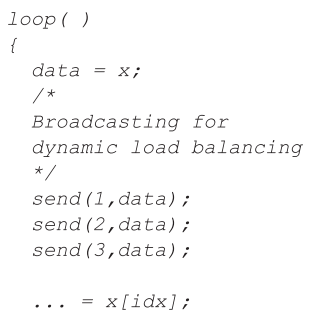

(a) Main

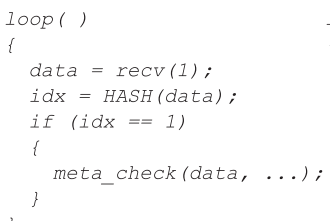

(b) Helper 1 ( tid = 1)

(c) Helper 2 ( tid = 2)

(d) Helper 3 ( tid = 3)

Fig. 2. Generated main and helper functions with dynamic load balancing using HASH.

how our algorithm replaces a call, communicates a predicate node, and places the computation of some inputs only in the helper thread. Statement " $x=$ goo $(x)$ " is a direct call, which must be preserved in the main thread and replaced in the helper with CHHTLookup, as shown in Figure 1(c). Next, consider the predicate node with the expression " $(x==1)$." Since a metafunction depends on this predicate, it must be preserved, and our algorithm is smart enough to communicate $\mathrm{x}$ and compute " $(x==1)$ " in the helper. Note that the main thread sends the predicate value using "send $(x)$ " and the helper thread receives the predicate value using " $x=\operatorname{recv}()$." Both operations occur at the same program point as the original definition of $x$. Finally, operations that support constant inputs of metafunctions are only placed in the helper. __FILE__ and ${ }_{-} L I N E_{--}$are two such examples. However, since $y$ is an input, we must communicate $x$ in order to compute $y$. In this case, our algorithm is smart enough not to send $x$ twice.

\subsection{Supporting Multiple Helpers with Load Balancing}

For our first approach to generate $N$ custom helpers, we use a static load balancing algorithm to create $N$ unique partitions from the $M$ set. Then, for each tid and $M_{t i d}$ from 1 to $N$, we use the CustomHelper algorithm to generate a custom helper. We have experimented with two static load balancing techniques to create the $N$ partitions of $M$ : round robin and random. For our round-robin approach, we traverse the CFG in DFS order and assign metafunctions to a partition using round robin. This is a simple approach that we have found to work well. We also considered random assignment. We use the same basic algorithm as round robin but place metafunctions into threads randomly. Random assignment can avoid imbalance caused by systematic interactions between the code structure and round-robin assignment. However, some applications perform poorly with static approaches.

Therefore, we also designed a dynamic load balancing algorithm in which the $M$ set is replicated to all the custom helpers instead of using a static partitioning. Since all helpers can work on any part of $M$, we dynamically assign work to each helper thread to balance its load. At compile time, our framework extracts metafunction codes from the main program and schedules them on all the customized helper threads. At runtime, we dynamically allow only one helper thread to execute the metafunction. Figure 2 shows the generated main function and helper functions for a loop with a large number of trip counts using dynamic load balancing techniques. As seen in Figure 2(a), the main thread sends data to all the helper threads. As seen in Figures 2(b), 2(c), and 2(d), metafunctions from the main program are extracted and scheduled on all the customized helper threads. In this example, we use a simple $H A S H$ function to select only one helper thread to execute the metafunction. However, other dynamic load balancing schemes are possible. 


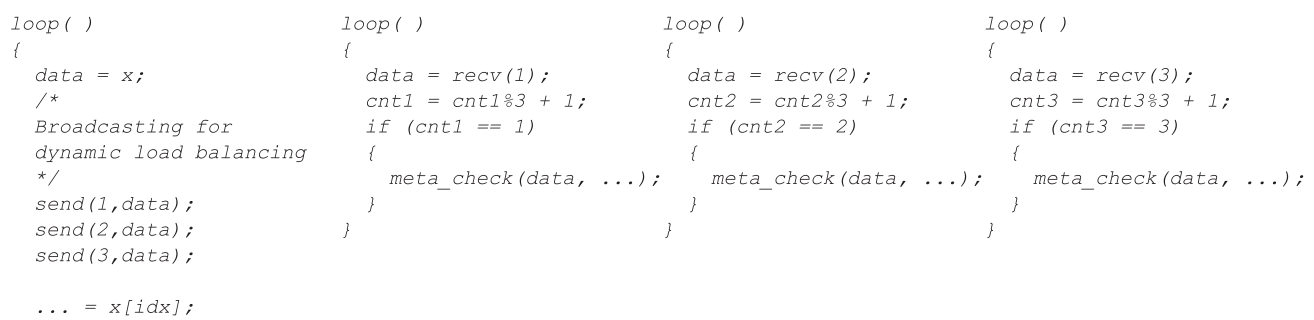
(a) Main
(b) Helper 1 ( tid = 1)
(c) Helper 2 ( tid = 2)
(d) Helper $3($ tid $=3$ )

Fig. 3. Generated main and helper functions with dynamic load balancing using counters.

Figure 3 shows another dynamic load balancing technique using local counters. As seen in Figure 3(a), main thread sends data to all the helper threads. As seen in Figures 3(b), 3(c), and 3(d), metafunctions from the main program are extracted and scheduled on all the customized helper threads in the same way as shown in Figure 3. However, we use a local counter (cnt1, cnt2, and cnt3) for each helper thread instead of a simple HASH function to select only one helper thread. Because a local counter of each helper thread is incremented in the same way, we dynamically allow only one helper thread to execute the metafunction.

While we expect to balance work more evenly on each helper, we also insert more instructions in the main thread by sending data to all the helper threads. With finegrained communications among all the threads, it will add more overheads into the main. In addition, by extracting all the metafunction codes and scheduling them on all the customized helper threads, it will add more communication for predicate nodes among the threads because predicate nodes should be communicated with all the helper threads. With static load balancing, because each metafunction is extracted and scheduled to a specific helper thread, predicate nodes were not communicated with all the helper threads. In Section 6, we show tradeoffs between static load balancing and dynamic load balancing due to these behaviors. Furthermore, in Section 6, we provide a couple of techniques to make dynamic load balancing techniques more efficient.

\section{METAFUNCTION LIBRARY REQUIREMENTS AND SUPPORT}

In the current formulation of our system, we require the programmer of the metafunction library to help in a few key ways. First, a list of all metafunctions must be provided to our compiler pass. This allows the compiler to identify metafunctions by name.

Second, the compiler is provided important knowledge regarding each metafunction. The compiler must know if it is an updating metafunction, UMF, or a validating metafunction, VMF. We assume that all VMFs can execute concurrently since they do not update MetaState. UMFs do change MetaState, and because of that, they potentially have a complex set of data dependence relationships to other metafunctions through the MetaState. For now, we keep it simple to make the compiler easy to implement. We assume that all UMFs must execute serially with respect to one another; however, this does not mean that they cannot be parallelized. If the MetaState is a single, shared structure, then all UMFs must be executed in a single thread, either main or one of the helpers, and all VMFs must serialize their execution with the nearest preceding and following UMF. If the MetaState is replicated across all helpers, then all UMFs must execute on each helper to construct the relevant state. The compiler enforces these properties in the generated code. 
Table II. Metafunctions Used by Mudflap

\begin{tabular}{|l|c|c|c|}
\hline Metafunction Name & Inputs & Insertion Point & Description \\
\hline -_mf_register & $\begin{array}{c}\text { Pointer to buffer, } \\
\text { size, location }\end{array}$ & $\begin{array}{c}\text { Any memory } \\
\text { allocation point }\end{array}$ & $\begin{array}{c}\text { Record allocated memory } \\
\text { region in a database }\end{array}$ \\
\hline -_mf_unregister & $\begin{array}{c}\text { Pointer to buffer, } \\
\text { location }\end{array}$ & $\begin{array}{c}\text { Any memory } \\
\text { deallocation point }\end{array}$ & $\begin{array}{c}\text { Remove previously allocated } \\
\text { region from database }\end{array}$ \\
\hline -_mf_check & $\begin{array}{c}\text { Pointer, } \\
\text { ref. size, location }\end{array}$ & $\begin{array}{c}\text { All memory } \\
\text { references }\end{array}$ & $\begin{array}{c}\text { Validate reference is for a } \\
\text { registered region of memory }\end{array}$ \\
\hline $\begin{array}{l}\text { Inlined software } \\
\text { cache lookup }\end{array}$ & $\begin{array}{c}\text { Pointer, } \\
\text { size }\end{array}$ & $\begin{array}{c}\text { All memory } \\
\text { references }\end{array}$ & $\begin{array}{c}\text { Checks a software cache to see if } \\
\text { reference has already been validated }\end{array}$ \\
\hline
\end{tabular}

Finally, the metafunction programmer must implement the metafunctions to work in a multithreaded program. Supporting concurrency within the library may not be too difficult in many cases. As long as the metafunctions use pass-by-value ${ }^{3}$ for all data extracted from the main program, data dependences will only occur between two UMF calls. Hence, protecting each metafunction with a transaction or a single lock would suffice to parallelize it; alternatively, converting all shared data to thread-local storage would also be sufficient. Of course, without careful attention, such approaches may underperform. It is likely that conversion of a serial library into a high-performing concurrent one will be nontrivial in the common case, but such conversion is beyond the scope of this work. We are not making it easy to support concurrency within each metafunction; rather, given a library that supports concurrency, our mechanism allows the exploitation of parallelism between the metafunctions and the original program.

\section{CASE STUDY: MUDFLAP}

To demonstrate the utility of our mechanisms, we selected the Mudflap library. Mudflap [Eigler 2003] is a set of passes and libraries integrated into GCC [Team 2008] to facilitate pointer-use checking. The Mudflap pass in GCC transparently adds an error checking code at the dereferencing of any pointer to validate the memory access against a database of allowed memory regions in the stack, heap, static variable section, and others.

Mudflap is not the most aggressive or accurate pointer-checking tool currently known [Nagarakatte et al. 2009, 2010; Hasabnis et al. 2012], but it is a production quality component of GCC, making it uniquely available and portable for research purposes. Since our goal is automatic helper thread extraction, Mudflap is a reasonable target for our work. We are not advocating Mudflap as a pointer-checking tool; rather, we are demonstrating our ability to parallelize it.

In this section, we consider how to support Mudflap parallelization in two ways. We will discuss how Mudflap maps into our automatic parallelization framework and discuss some synergistic benefits we discovered. Also, we will describe our strategy for manually parallelizing it. In the evaluation, we will compare these two approaches.

\subsection{Mapping Mudflap to Our Framework}

Table II shows the metafunctions used by the Mudflap algorithm. _-_mf_register is an updating metafunction added at every memory allocation point to record a new memory region that can be accessed by the program. Such memory allocation points include malloc, stack allocation, and global variables. _.mf_unregister is an updating metafunction that is inserted at all deallocation points. And __mf_check is validating

\footnotetext{
${ }^{3}$ This is in contrast to pass-by-reference, which could lead to data races between the helper thread and the main thread. Data races would violate our initial assumptions, presented in Section 2.
} 


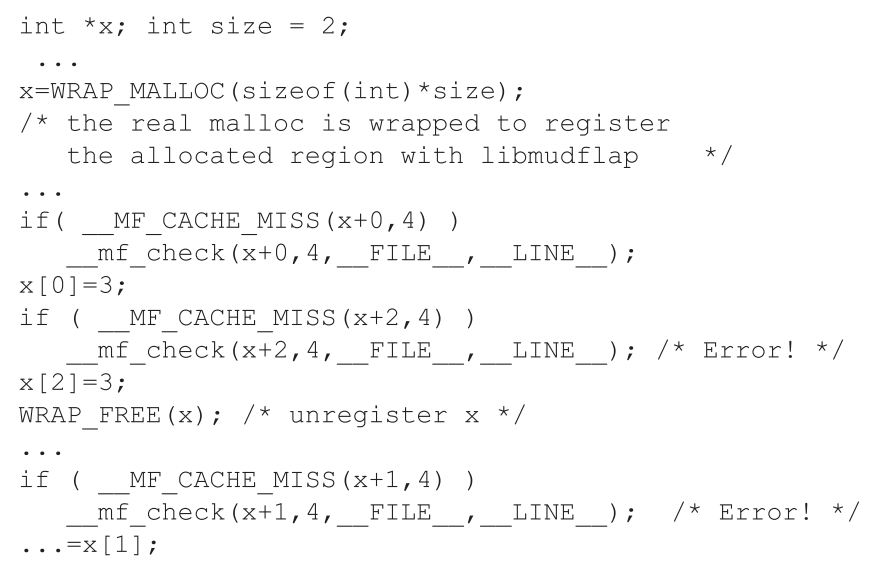

Fig. 4. Example code with Mudflap annotation.

metafunction that checks if a memory reference falls within an allocated region. Figure 4 provides an example of a program annotated with Mudflap's metafunctions.

We have studied the behavior of Mudflap to determine the appropriate strategy for parallelizing these metafunctions. Since __mf_register and __mf_unregister are relatively infrequent and since the database they create is relatively small, they are always replicated across all helpers. _-_mf_check, on the other hand, is very frequent and must be balanced across the helpers to provide scalable performance improvement. Table I from Section 3 backs up these decisions. GCC provides a library, called libmudflap, that implements these metafunctions. We modified this library to support a parallel implementation. In particular, we changed the code so that a database could be created and maintained per thread using thread local storage. This only required changing a few lines of code.

Since the C library is not compiled with Mudflap annotations, library functions that manipulate pointers must be handled as a special case. Libmudflap already provides wrapper functions for many libC functions that allocate memory. For the case of malloc and free, a wrapper function performs the register or unregister, respectively, on the allocated or freed region. We modified all such wrapper functions manually to provide a customized helper and added them in the CHHT.

Benefits for Mudflap. Our framework is expected to provide several useful benefits for Mudflap. Looking at the inputs for each metafunction, it is clear that two of the inputs are likely constants: size and location. These inputs will be extracted directly into the custom helper. The pointer must be communicated, but if it is used in multiple metafunctions, it may only need to be communicated once. Finally, the inlined software cache look-up is directly inserted into the program to prevent redundant calls to _-_mf_check. These inlined codes add significant instruction and control flow overhead, and all of these can be moved to the helper.

Our framework also provided some unexpected benefits. Local aggregates need to be registered with Mudflap. However, when a helper is created, we initially copy all local variable declarations into the helper. Since the helper's copy is not the same memory location as the one in the main program, the local copy in the helper need not be registered with Mudflap. But we found it beneficial to do so. By registering the helper's local aggregate, many metafunctions could be executed using the local aggregate without ever receiving input from the original function. An out-of-bounds check in the helper on a copy is equivalent to a check in the original function. This optimization reduced communication and thereby lightened the load on the main program. N.B. We still 


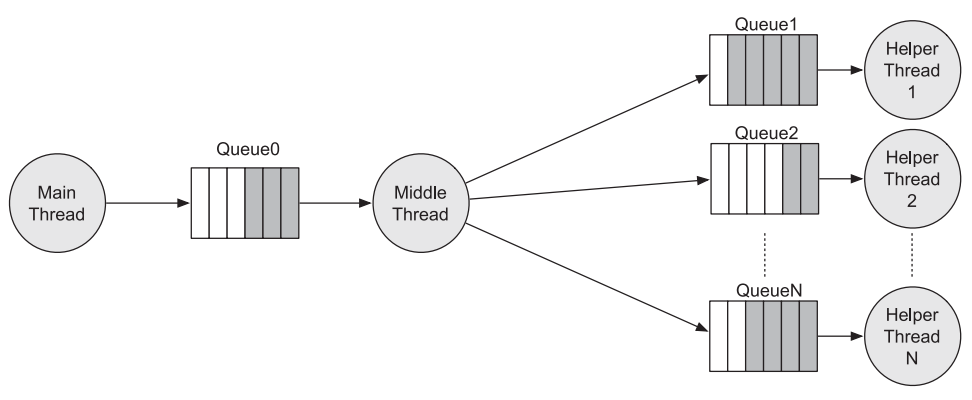

Fig. 5. Mudflap manual parallelization.

register the address of the original aggregate from the main program just in case its address is ever taken.

\subsection{Manual Parallelization}

In order to evaluate how well our automatic technique works, we also manually parallelized Mudflap for comparison. In this implementation, we just rewrote the metafunctions to exploit parallelism explicitly.

The metafunctions implement a pipelined architecture as shown in Figure 5. For each metafunction in Mudflap, we created a parallel version that packs up its input and enqueues them to Queue0. Each metafunction inserts a special header at the beginning of its input so that it can be parsed properly by the middle thread. Also, we modified __mf_check to include the software cache look-up. This modification significantly reduces the code injected into the main program. Also, to make _-_mf_check a little more efficient, we omitted the debugging location information. For every pointeruse check, we insert this alternative implementation of __mf_check.

The middle thread parses the contents of Queue0 and decides how to handle the metafunction. It must send __mf_register and __mf_unregister requests to all helpers. For _-_mf_check requests, it applies a load balancing heuristic and sends the request to one of the helper threads for completion. Because the performance of the system is very sensitive to the processing rate of the middle thread, the load balancing heuristics are kept simple and fast. We considered two dynamic load balancing techniques: address-based balancing and round robin.

Parallelization 1: Address Indexing. Because the helpers use a software cache to filter out redundant checks, we prefer to send redundant checks to the same core so they will be filtered quickly. We use a simple hash function on the input address to select a queue.

Parallelization 2: Round Robin. We also evaluate the middle thread using a round-robin method to select a helper thread. Using this approach, allocation is more uniform, and the middle thread is less likely to block on a full queue.

Our round-robin algorithm sends eight consecutive operations to the same helper. By sending eight at a time, we exploit locality in the access stream; consecutive checks are more likely to refer to the same object in the Mudflap database. We determined a chunk size of eight through experimentation.

\section{EVALUATION}

\subsection{Setup}

We have evaluated our design on SESC [Renau et al. 2004], an event-driven performance simulator that accurately models CMP architectures. Table III shows the pertinent details of the simulated architecture. The core and the L1 and L2 caches 
Table III. Architecture Simulated

All cycle counts are in processor cycles.

\begin{tabular}{|c|c|}
\hline \multicolumn{2}{|l|}{ 8-Core CMP } \\
\hline Frequency $3.2 \mathrm{GHz}$ & ROB 126 \\
\hline Fetch width 6 & I-window 68 \\
\hline Issue width 3 & LD/ST queue 48/42 \\
\hline Retire width 3 & Mem/Int/Fp unit 1/2/1 \\
\hline Branch predictor: & Hybrid GShare \& Local \\
\hline \multicolumn{2}{|l|}{ Mispred. Penalty 14 cycles } \\
\hline \multicolumn{2}{|l|}{ BTB 2K, 2-way } \\
\hline Private L1 Cache: & Private L2 Cache: \\
\hline Size $64 \mathrm{~KB}$, assoc 4 & Size $2 \mathrm{MB}$, assoc 8 \\
\hline Line 64B & Line $64 \mathrm{~B}$ \\
\hline \multirow[t]{5}{*}{ Latency 2 cycles } & Latency 9 cycles \\
\hline & Protocol MESI \\
\hline & Memory: \\
\hline & Latency 300 cycles \\
\hline & Bandwidth 25.6GB/s \\
\hline
\end{tabular}

are common for all architectures we evaluated. The core is an aggressive three-issue superscalar processor. We model a cache coherent CMP design with private L1 and L2 caches. Coherence is supported at the L2 cache over a ring interconnect.

For this study, we use highly efficient hardware queues for communication between threads. We assume one cycle to read/write from/to the queue. Also, we used $4 \mathrm{~KB}$ for each hardware buffer. We used hardware queues rather than software queues to showcase the effectiveness of our parallelization strategy independent of queue implementation. Software queues need careful tuning in both an application-specific and architectural-specific way [Jablin et al. 2010]. Prior work demonstrated that software queues perform poorly in a manually parallelized Mudflap implementation [Lee et al. 2011]; however, the key bottleneck is overhead per queue operation, not the delay between enqueue and dequeue. With sufficient tuning, it may be possible to achieve high performance with fully software queues. Since we have not carried out that tuning effort, we pick a low-overhead hardware queue. We also compare to HAQu [Lee et al. 2011], a hardware-accelerated queue, in Section 6.7, which uses the on-chip interconnect to communicate data.

Table IV shows the application binaries we study. By default, we target eight cores, but some binaries are compiled for 16 . That is denoted with a 16 at the end of their name. For all of our application binaries, we used GCC 4.5 targeted to the Mips32 architecture. All of our compiler algorithms described in this article were implemented in GCC. To accurately compare the performance of different binaries, simply timing a fixed number of instructions cannot be used. Instead, "simulation markers" are inserted in the code of each binary, and simulations are run for a given number of markers. After skipping the initialization, a certain number of markers are executed so that the binary graduates from 100 to 500 million instructions.

We evaluated our approach on a subset of the SPEC CPU Integer 2000 application suite. We excluded applications that did not work with our cross-compiler (vortex and eon), and we excluded gcc, crafty, and perlbench because the Mflap binary (Mudflap out-of-the-box) encountered errors during execution on our simulator. 
Table IV. Configurations and Parallelizations

\begin{tabular}{|l|l|}
\hline Name & \multicolumn{1}{|c|}{ Description } \\
\hline Base & No Mudflap,-O3 \\
Mflap & Base + Mudflap \\
A-Random & Automatic Parallelization with static Random Assignment \\
A-RoundRobin[16] & Automatic Parallelization with static Round-robin Assignment \\
DLB-Hash[16] & Automatic Parallelization with dynamic load balance using Hash \\
DLB-Counter & Automatic Parallelization with dynamic load balance using \\
& Counter \\
DLB-Pipe & Automatic Parallelization with dynamic load balance using \\
& Pipelined Communication \\
M-Index & Manual Parallelization with Address Indexing Assignment \\
M-RoundRobin & Manual Parallelization with Round-robin Assignment \\
BHE4 & Baseline Helper Extraction with 4 threads \\
BHE8 & Baseline Helper Extraction with 8 threads \\
\hline
\end{tabular}

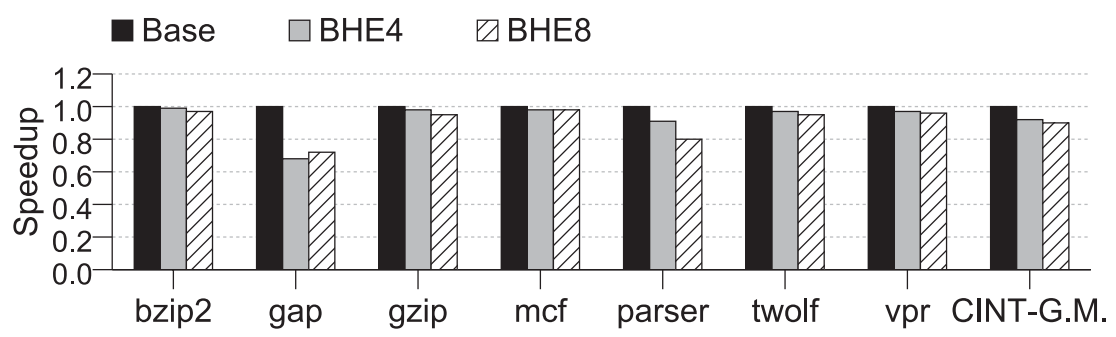

Fig. 6. Scalability of Base Helper Extraction with four cores and eight cores.

\subsection{Baseline Helper Extraction}

In this section, we evaluate the scalability of our BaseHelperThread extraction algorithm with several SPECint applications on a four-core and eight-core CMP. Figure 6 shows the speedup over Base. CINT-G.M is the geometric mean normalized to Base over all of the applications. On average, our proposal performs $8 \%$ worse than Base for both four-core and eight-core CMP configurations. However, gap loses $30 \%$ and parser loses $20 \%$, while the others only lose between $2 \%$ and $5 \%$. That is, Helper extraction is scalable for eight cores with only $2 \%$ to $5 \%$ loss. gap and parser show a larger slowdown compared to the others because they have a large number of function calls. The calls alone are not expensive, but in order to match the control path of the main thread, they require frequent communication of predicates, which adds a lot of overhead to the main. With this overhead, gap and parser are limited in the parallelism they can support.

\subsection{Custom Helper Extraction}

In this section, we evaluate several characteristics of the parallelized Mudflap metafunctions for several SPECint applications on the eight-core CMP. Figure 7 shows the speedup over Mflap. For this evaluation, we use empty _-mf_register, __mf_unregister, and __mf_check metafunctions to show the quality of parallelization without the effects of runtime load imbalance. In addition, we compare our two static algorithms for load balancing. Looking at the overall geometric mean, our design achieves $4.5 \times$ speedup with random assignment and $4.6 \times$ speedup with round-robin assignment. This suggests that our extracted helpers are quite efficient. Furthermore, the performance has only been degraded by $26 \%$ over the results in Figure 6.

Figure 8 shows the speedups compared to Mflap running the full Mudflap implementation using both the automatic parallelization with static load balancing and 


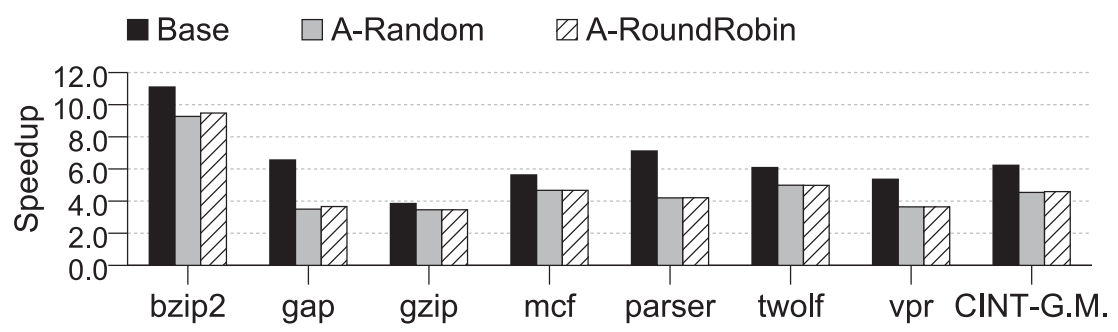

Fig. 7. Speedups of parallelized Mudflap with empty runtime functions.

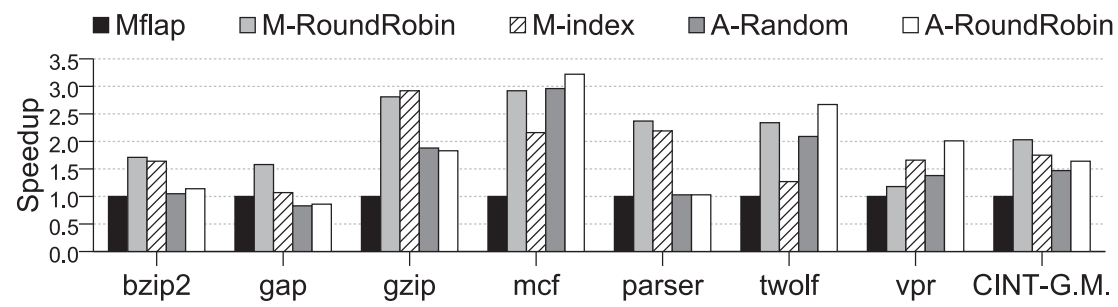

Fig. 8. Speedups of parallelized Mudflap.

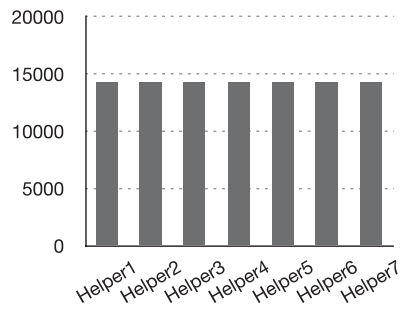

(a) M-RoundRobin

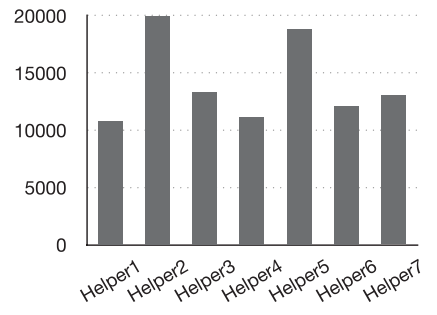

(b) M-Index

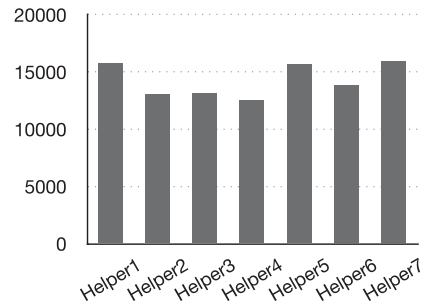

(c) A-RoundRobin

Fig. 9. The number of __mf_checks in each helper thread on eight-core CMP (gap).

the manual parallelization. Overall, looking at the geometric mean, the automatically parallelized code is only $19 \%$ slower than the best manual technique. Furthermore, the automatic technique is performing competitively; mcf, twolf, and vpr achieve their highest performance using A-RoundRobin. mcf, twolf, and vpr achieve their advantage because their workloads are relatively balanced, and they perform well using a static load balancing approach. This case highlights the importance of automatic metafunction extraction. However, bzip2, gap, gzip, and parser are underperformers. gzip suffers from poor load balance; the manual cases achieve speedups within $25 \%$ of Base because of their aggressive load balancing strategies. bzip2 also suffers primarily from poor load balance. Finally, gap and parser perform poorly due to a combination of factors: poor load balance and the significant communication overhead needed to support function calls.

\subsection{Dynamic Load Balancing}

In Section 6.3, we speculated that imbalance hurt the performance of some applications. In order to estimate the overhead due to imbalance, we measured the number of metafunctions executed in each helper thread. Figures 9 and 10 show the number of __mf_checks executed in each dynamic helper thread with various load balancing techniques. 


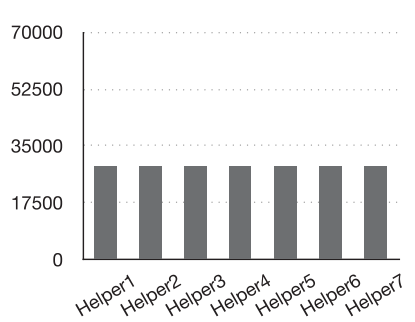

(a) M-RoundRobin

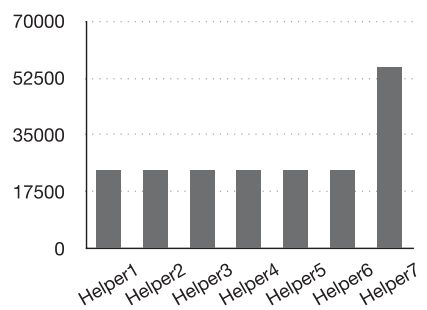

(b) M-Index

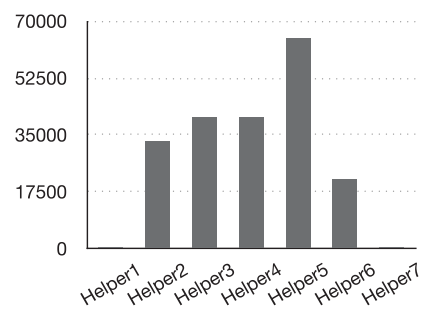

(c) A-RoundRobin

Fig. 10. The number of __mf_checks in each helper thread on eight-core CMP (gzip).

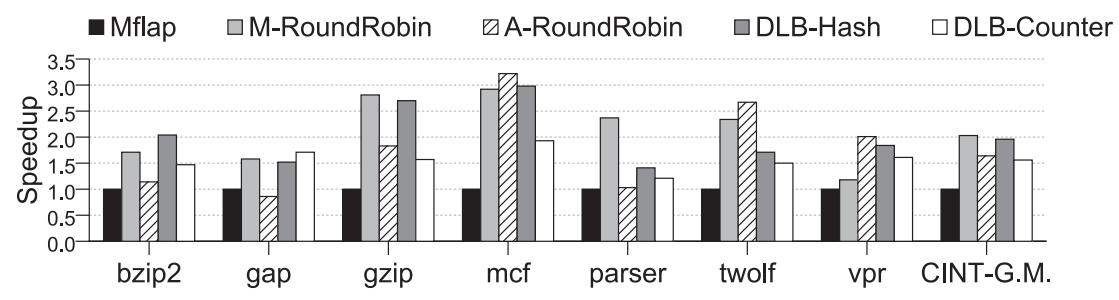

Fig. 11. Speedups of parallelized Mudflap with various load balancing.

Figure 9 shows the work distribution across the threads in gap. While manual parallelization using round robin (Figure 9(a)) has an equally distributed workload, manual parallelization using address indexing (Figure 9(b)) and automatic parallelization using static round robin (Figure 9(c)) have an imbalanced workload. This supports the data in Figure 8 that shows better performance with M-RoundRobin than M-index and A-RoundRobin. Figure 10 shows the workload for each thread in gzip. In gzip, M-RoundRobin (Figure 10(a)) and M-index (Figure 10(b)) are well balanced. However, A-RoundRobin (Figure 10(c)) shows an imbalanced workload for each thread. As a result, Figure 8 shows that gzip performs better with M-RoundRobin and M-index than A-RoundRobin. Even though helper 7 in Figure 10(b) has a large number of _-_mf_checks compared to its other helpers, its workload is similar because of memoization techniques that occur within __mf_check.

Figure 11 shows the speedups for several static and dynamic load balancing techniques. In the figure, we compared dynamic load balancing techniques, labeled as DLBHash and DLB-Counter, to M-RoundRobin and A-RoundRobin normalized to Mflap. On average, DLB-Hash achieves a $1.96 \times$ speedup over Mflap. DLB-Counter achieves a $1.56 \times$ speedup over Mflap. On average, DLB-Hash is competitive to M-RoundRobin for manual parallelization.

In bzip2, gap, and gzip, DLB-Hash is better than M-RoundRobin. DLB-Hash exploits similar benefits as M-Index, which works well for bzip2 and gzip. DLB-Counter offers benefits similar to M-RoundRobin for gap. As described in Section 6.3, mcf, twolf, and vpr achieve their highest performance using A-RoundRobin. Because A-RoundRobin evenly assigns work to each helper in mcf, twolf, and vpr, they perform very well. However, DLB-Hash and DLB-Counter degrade their performance because of additional communication overhead among threads.

Looking at the combined benefits of A-RoundRobin, DLB-Hash, and DLB-Counter, all but parser perform well with the automatic techniques. gzip does show a small slowdown with automatic parallelization against manual parallelization, but it is ignorable. For parser, we see that DLB-Hash performs better than A-RoundRobin, but DLB-Hash is much worse than manual parallelization. To better understand why, we measured 


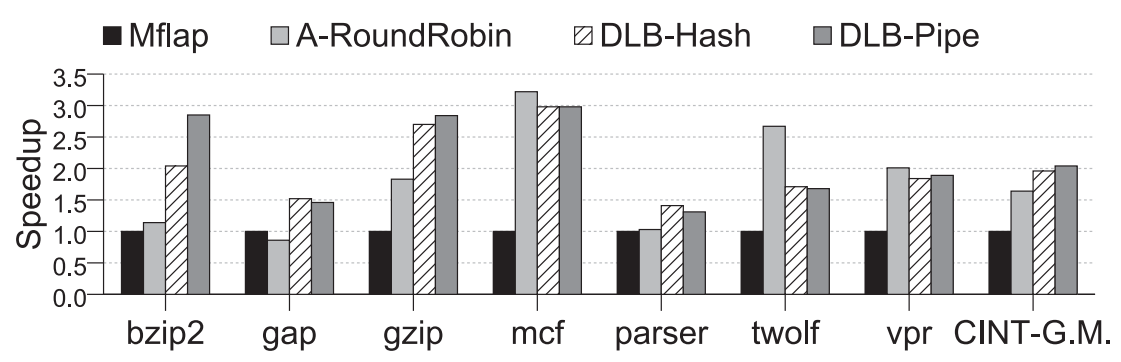

Fig. 12. Speedups of pipelining communications compared to other parallelizations.

the dynamic instruction count of parser using the Base Helper Extraction algorithm without any additional instrumentation for Mudflap. Even for this case, parser already has as many instructions as M-RoundRobin due to high communication overhead with its helpers. Hence, our approach is simply uncompetitive for parser. In order to get better performance for applications like parser, reducing communication or performing it more efficiently is required.

\subsection{Optimizations}

In this section, we provide and evaluate a couple of parallelization techniques to get better performance for each application. In addition, we also evaluate our framework on a 16-core system to show the scalability of our automatic parallelization framework.

Pipelining Communication. By measuring the number of dynamic instructions in DLB-Hash and DLB-Counter, we found that a large number of instructions were devoted to communication between the main thread and its helpers. This largely stems from a common communication idiom in which the main sends the same data to all threads using a separate send operation. This places communication on the critical path of the main, which is bad for performance.

To reduce this overhead, we pipelined the communication. That is, the main sends data to one helper, and the helper forwards it along to the others. Each thread is responsible to send the data to one other thread until all threads have received the data. Figure 12 shows the speedup using pipelined communication. bzip2 and gzip both benefit. This is not surprising since bzip2, gap, and gzip achieved the best performance with DLB. Compared to the other applications, bzip2 is able to get the best speedup because its helpers are evenly balanced, which ensures timely communication through the pipe. On the other hand, if any single thread is delayed too much, it delays communication to the next thread, which leads to more imbalance. Such behavior explains why gap slows down a little bit even though its main thread has less work.

Combining Static and Dynamic Load Balance. We have experimented with combinations of static and dynamic load balancing to get the best of both. Because parser did not perform well with automatic parallelization, we evaluate the technique with parser. Using an edge profile, we found the static locations of the most executed metafunctions. For these high-frequency metafunctions, we use dynamic load balancing; we use static load balancing for the rest. As a result, we achieve a $6 \%$ speedup over DLB-Hash in parser.

On a 16-Core CMP. We evaluate the scalability of our static and dynamic load balancing techniques on an eight-core and a 16-core CMP. Figure 13 compares the performance of A-RoundRobin (eight cores), DLB-Hash (eight cores), A-RoundRobin16 (16 cores), and DLB-Hash16 (16 cores). Interestingly, bzip2, gap, gzip, mcf, and twolf all achieve better performance on a 16-core CMP for one of the policies. However, parser and vpr degrade performance a little when using more cores. Overall, there 


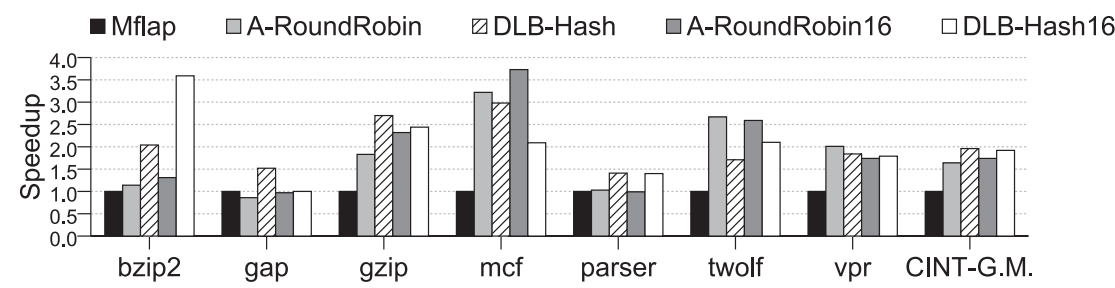

Fig. 13. Scalability of automatic parallelization framework with eight cores and 16 cores.

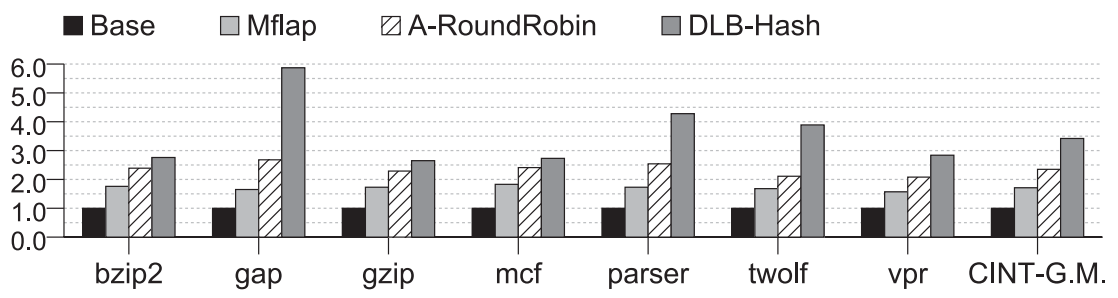

Fig. 14. Binary size.

are two interesting trends. First, A-RoundRobin16 tends to improve or be about the same as A-RoundRobin. This is because of its low overhead, requiring little coordination between the main thread and the helpers. On the other hand, DLB-Hash16 offers a high speedup over DLB-Hash in the case of bzip2. Since bzip2 suffers from significant load imbalance, the extra overhead is overcome by utilization of additional cores. However, in cases where there is little load imbalance or insufficient work for 16 cores, the added communication overhead is severe and degrades performance. Hence, gap, gzip, mcf, and vpr all perform worse using DLB-Hash16.

In summary, A-RoundRobin16 is more predictable when scaled in that it usually preserves or improves performance; however, DLB-Hash16 can offer large scaling benefits when load imbalance is in a bottleneck.

\subsection{Overheads}

We have also measured some key overheads in our system. Figure 14 shows the relative size of the binary for four configurations: Base, Mflap, A-RoundRobin, and DLB-Hash. On average, Mflap is almost $1.71 \times$ bigger than Base. Our technique adds additional overhead due to Custom Helper creation. This overhead is $38 \%$ for A-RoundRobin and $100 \%$ for DLB-Hash, on average, over Mflap.

We also measured the number of dynamic instructions in the main thread for Base, Mflap, M-RoundRobin, A-RoundRobin, and DLB-Hash. We can see that the parallelized versions do significantly reduce the overhead in the main thread. M-RoundRobin slightly edges out A-RoundRobin for two reasons: (1) the main program only communicates with one other thread, which keeps its communication overhead low; and (2) we have eliminated debugging information from the _-_mf_check metafunction for manual parallelization. In contrast, A-RoundRobin must send inputs for __mf_register and _-_mf_unregister to all custom helpers. Furthermore, other data, like control predicates, also get sent to multiple helpers. Currently, we do nothing to reduce this overhead, but we plan to address these communication inefficiencies in future work.

\subsection{Comparison to Hardware Accelerated Queues}

In order to better understand the importance of custom hardware queues, we include a comparison to $\mathrm{HAQu}$ [Lee et al. 2011], a hardware accelerated queue that uses 


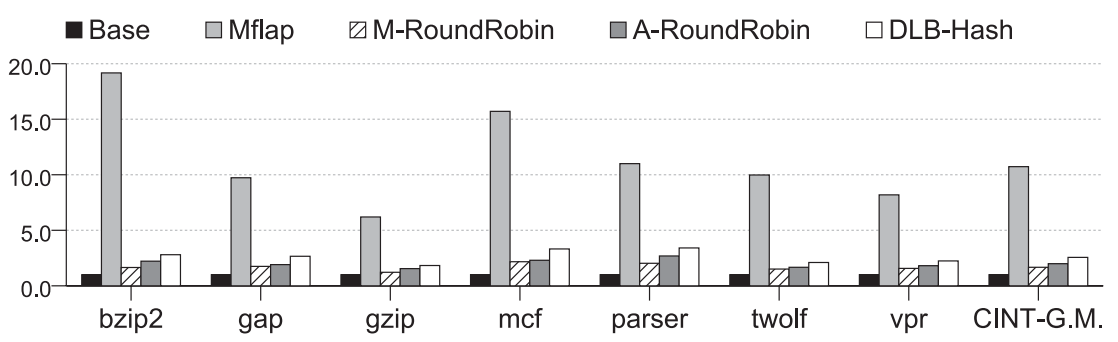

Fig. 15. The main thread's dynamic instruction counts.

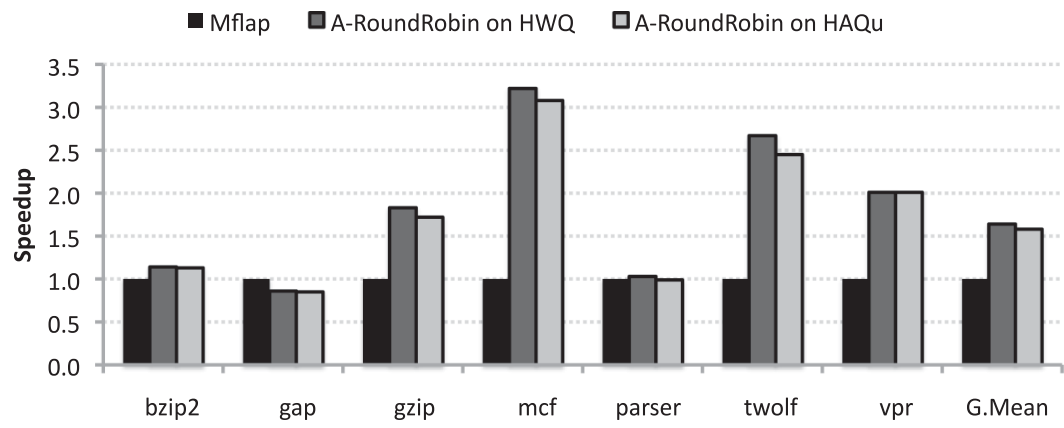

(a) Static load balancing techniques.

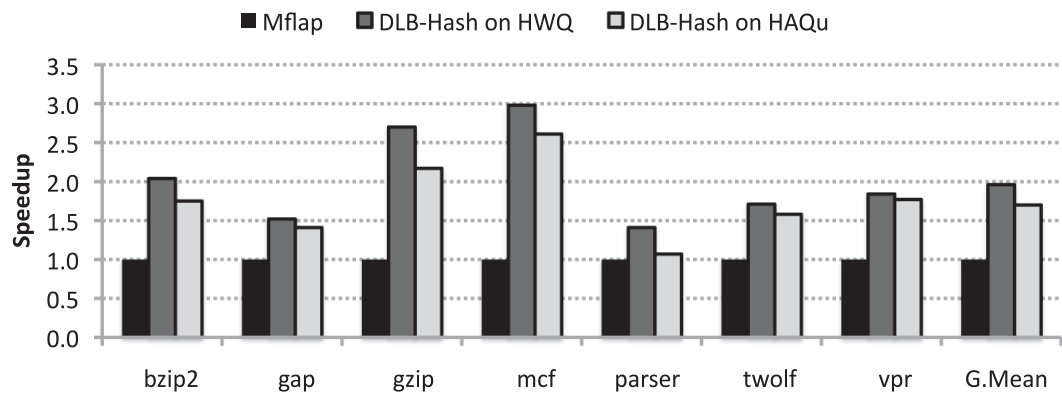

(b) Dynamic load balancing techniques.

Fig. 16. Performance comparison of custom hardware queues to HAQu.

the on-chip interconnect for all point-to-point communication. Hence, the latency of communication is lengthened when using HAQu since all data is moved through the interconnect. For the HAQu runs, we replace the hardware queues with the support proposed in Lee et al. [2011]: a Queue Functional Unit per core with support for two queue operations per cycle. All other parameters remain the same.

Figure 16 shows a comparison for static load balancing and dynamic load balancing techniques. Figure 16(a) shows that using HAQu results in a minor performance drop for static load balancing. However, dynamic techniques suffer a larger drop, shown in Figure 16(b). Since the dynamic techniques require greater communication throughput, they suffer more from using the on-chip interconnect. Of course, improvements in interconnect technology could render these differences moot. 


\section{RELATED WORK}

Helper Threads. Many works have improved single-thread performance by using helper threads [Luk 2001; Roth and Sohi 2001; Tiwari et al. 2010; Wang et al. 2004; Xekalakis et al. 2009]. In Luk [2001] and Roth and Sohi [2001], to tolerate memory bottlenecks, the helper threads perform pre-execution with idle hardware resources. Xekalakis et al. [2009] combined helper threads with Thread-Level Speculation and Runahead execution. Tiwari et al. [2010] used a helper thread to accelerate memory management.

Parallelizing Security Metafunctions. While many security protection mechanisms have been proposed, few have investigated exploiting parallelism between the application and its security metafunctions, which is the focus of this proposal. Nightingale et al. [2008] proposed Speck, which exploits speculative parallelism between the application being protected with its security metafunctions. Shetty et al. [2004] and Kharbutli et al. [2006] parallelize memory management functionality for increased security. Plakal and Fischer [2000] proposed parallelizing garbage collection using program slices on multiprocessors. However, they did not implement the slices; rather, they only evaluated the potential.

Thread-Level Speculation. One alternative way to exploit parallelism between the application and its metafunctions is to use thread-level speculation (TLS). TLS allows frequent data sharing at low cost, but without a guarantee that the speculative work will be useful. Examples of work in bug detection with TLS include Oplinger and Lam [2002], Zhou et al. [2004b], and Kelsey et al. [2009]. All studies employ thread-level speculation to hide the overhead of program checking. It has been applied in security by Speck [Nightingale et al. 2008], as discussed previously.

FastTrack [Kelsey et al. 2009] is especially relevant since it also considers parallelization of Mudflap. Similar to our approach, its goal is to make the main program execute without metafunction overhead. FastTrack runs a fast version of the code without checks in a separate process in parallel with a full version of the code with checks. The full version of the code verifies that the fast version performed the correct work. To achieve high performance, if the fast code finishes a code region early, it launches the next region with both a fast and a full version using its own state as the seed. As a result, it's able to run ahead and overlap the execution of the checking code. FastTrack is compelling in that no knowledge of the metafunction is needed to parallelize its execution. However, since it retains the illusion of being a single-threaded program, overhead is incurred to detect and recover from mis-speculations. Furthermore, it may not be able to exploit relationships among metafunctions as effectively since some dependence violations may not alter the meaning of the program. Comparing our reported results to theirs, our approach outperforms FastTrack on eight cores for applications evaluated in both studies. However, their results are measured on a real system while ours are simulated.

Memory Bugs. Detection of memory bugs also can be performed statically by utilizing explicit model checking [Musuvathi et al. 2002; Stern and Dill 1995] and program analysis [Choi et al. 2002; Engler and Ashcraft 2003; Hallem et al. 2002]. As software becomes more complex and diverse, users increasingly rely on runtime bug detection by instrumenting the code with checks, such as in Purify [Rational Software 2006], Valgrind [Seward 2004; Seward and Nethercote 2005], Intel thread checker [KAI-Intel Corporation 2004], DIDUCE [Hangal and Lam 2002], Eraser [Savage et al. 1997], CCured [Necula et al. 2002], and others [Austin et al. 1994; Cowan et al. 1998; Loginov et al. 2001; Patil and Fischer 1997, 1995]. Such instrumentation typically introduces large performance overheads because instrumented memory references (loads and stores) are executed often, and execution of the instrumentation code is interleaved with normal program execution. 
Hardware support for detecting memory bugs or to support debugging has been proposed, such as iWatcher [Zhou et al. 2004b], AccMon [Zhou et al. 2004a], SafeMem [Qin et al. 2005], DISE [Corliss et al. 2005], and the scheme proposed by Oplinger and Lam [2002]. All three schemes (iWatcher, AccMon, and DISE) interleave application execution with bug checking, address matching, or debugging functions. In contrast, our helper computing mechanisms attempt to exploit parallelism between the helpers and the main application execution.

\section{DISCUSSION AND FUTURE WORK}

Our system works well on fine-grained metafunctions that extract limited data from the main program. Pointer checking is well suited for our system since it was the inspiration for our approach. However, other metafunctions that work in a similar way can be supported. We would only need to inform the compiler of the metafunctions and their dependences to enable correct parallelization. For these cases, simplifying the steps for supporting new metafunction libraries is a key focus of future work.

In other cases, we expect that changes are needed to our approach. For example, in the security domain, metafunctions are used to detect an attack before the system is compromised. If such metafunctions are executed asynchronously with the main thread, an attack may occur before the metafunction can detect the problem. Such a scenario is unacceptable. Hence, the rules for metafunction parallelization will need additional context or constraints that force them to execute synchronously. However, since this may force the main thread to wait on the helper, we would expect some performance degradation if this behavior were frequent. Speculative execution [Nightingale et al. 2008; Kelsey et al. 2009] may be helpful for avoiding such performance degradation.

Finally, since our current approach primarily focused on reducing latency, the power consumption of our approach was not a big concern. When we create helper threads that track the control flow of the main program, a significant amount of work is replicated across the main thread and the helper threads. As power and energy are a significant concern for computer systems at all scales, additional analysis is needed to fully characterize the power impact of our approach and look for ways to optimize it.

\section{CONCLUSION}

In this work, we consider an automatic thread extraction technique for removing finegrained checks from a main application and scheduling them on helper threads. In this way, we can leverage the resources available on a CMP to reduce the latency of fine-grained checking codes. In addition, we explore a variety of techniques to improve performance. We evaluate our parallelization strategy on Mudflap, a pointer-use checking tool in GCC. To show the benefits of our techniques, we compare them to a manually parallelized version of Mudflap. We run our experiments on an architectural simulator with support for fast queueing operations.

On a subset of SPECint 2000, our automatically parallelized code using static load balancing is only $19 \%$ slower, on average, than the manually parallelized version on a simulated eight-core system. Furthermore, three applications achieve better speedups using our algorithms than with the manual approach. Our approach introduces very little overhead in the main program-it is kept under $100 \%$, which is more than a $5.3 \times$ reduction compared to serial Mudflap. We evaluated our framework on 16-core systems to better understand the scalability of our framework. We do see an improvement in performance, on average, for static and dynamic load balancing when moving from eight to 16 cores. Hence, our approach offers some scalability, especially in applications with large overheads, like bzip2. However, much of the remaining overhead is instrumentation added to the main and cannot be eliminated through threading. 
In conclusion, our automatically parallelized Mudflap library using a combination of static and dynamic load balancing is competitive, on average, with the manually parallelized version of it. Many challenging problems remain in this area. First, can the relationships among metafunctions be analyzed automatically for use by our infrastructure? Second, we need to identify the features needed to support a wider variety of metafunctions. Finally, eliminating the remaining overheads in the main and optimizing the power consumption of the approach need further investigation. The answer to some of these challenges may lie in techniques like speculative threading [Kelsey et al. 2009], better dependence analysis, and improved architectural features for helper threading [Lee et al. 2011].

\section{REFERENCES}

Austin, T. M., Breach, S. E., AND Sohi, G. S. 1994. Efficient detection of all pointer and array access errors. In Proceedings of the ACM SIGPLAN Conference on Programming Language Design and Implementation.

Choi, J., Lee, K., Loginov, A., O'Callahan, R., Sarkar, V., and Sridharan, M. 2002. Efficient and precise datarace detection for multithreaded object-oriented programs. In Proceedings of ACM SIGPLAN 2002 Conference on Programming Language Design and Implementation.

Corliss, M., Lewis, E., AND Roth, A. 2005. Low-overhead interactive debugging using DISE. In Proceedings of the International Symposium on High Performance Computer Architecture.

Cowan, C., Pu, C., Maier, D., Walpole, J., Bakke, P., Beattie, S., Grier, A., Wagle, P., Zhang, Q., And Hinton, H. 1998. StackGuard: Automatic adaptive detection and prevention of buffer-overflow attacks. In Proceedings of the 7th USENIX Security Symposium. 63-78.

Eigler, F. 2003. Mudflap: Pointer use checking for C/C++. In Proceedings of the GCC Developers Summit.

Engler, D. AND AshCRAFT, K. 2003. RacerX: Effective, static detection of race conditions and deadlocks. In Proceedings of the 19th ACM Symposium on Operating Systems Principles.

Ferrante, J., Ottenstein, K. J., and Warren, J. D. 1987. The program dependence graph and its use in optimization. ACM Trans. Program. Lang. Syst. 9, 3, 319-349.

Hallem, S., Chelf, B., Xie, Y., And Engler, D. 2002. A system and language for building system-specific, static analyses. In Proceedings of the ACM SIGPLAN Conference on Programming Language Design and Implementation.

Hangal, S. AND Lam, M. S. 2002. Tracking down software bugs using automatic anomaly detection. In Proceedings of the International Conference on Software Engineering.

Hasabnis, N., Misra, A., And Sekar, R. 2012. Light-weight bounds checking. In Proceedings of the 10th International Symposium on Code Generation and Optimization (CGO'12). ACM.

Jablin, T., Zhang, Y., Jablin, J., HuAng, J., Kim, H., AND August, D. 2010. Liberty queues for epic architectures. In $E P I C-8$.

KAI-Intel CoRPoration. 2004. Intel Thread Checker. http://developer.intel.com/software/products/threading/ tcwin.

Kelsey, K., Bai, T., Ding, C., AND Zhang, C. 2009. Fast track: A software system for speculative program optimization. In Proceedings of the 2009 International Symposium on Code Generation and Optimization.

Kharbutli, M., Jiang, X., Venkataramani, G., Solihin, Y., and Prvulovic, M. 2006. Comprehensively and efficiently protecting the heap. In Proceedings of the International Symposium for Programming Languages and Operating Systems.

Lee, S., Tiwari, D., Solihin, Y., And Tuck, J. 2011. HAQu: Hardware Accelerated Queueing for fine-grained threading on a chip multiprocessor. In Proceedings of the 17th International Symposium on HighPerformance Computer Architecture.

Loginov, A., Yong, S. H., Horwitz, S., And Reps, T. 2001. Debugging via run-time type checking. Lecture Notes in Computer Science, vol. 2029.

LuK, C.-K. 2001. Tolerating memory latency through software-controlled pre-execution in simultaneous multithreading processors. In Proceedings of the 28th International Symposium on Computer Architecture.

Musuvathi, M., Park, D., Chou, A., Engler, D. R., And Dill, D. L. 2002. CMC: A pragmatic approach to model checking real code. In Proceedings of the Fifth Symposium on Operating Systems Design and Implementation.

Nagarakatte, S., Zhao, J., Martin, M. M. K., and Zdancewic, S. 2009. SoftBound: Highly compatible and complete spatial memory safety for c. PLDI, 245-258. 
Nagarakatte, S., Zhao, J., Martin, M. M. K., and Zdancewic, S. 2010. CETS: Compiler enforced temporal safety for C. ISMM, 31-40.

Necula, G. C., McPeak, S., And Weimer, W. 2002. CCured: Type-safe retrofitting of legacy code. In Proceedings of the Symposium on Principles of Programming Languages.

Newsome, J. AND Song, D. 2005. Dynamic taint analysis for automatic detection, analysis, and signature generation of exploits on commodity software. In Proceedings of the 12 th Annual Network and Distributed System Security Symposium (NDSS).

Nightingale, E. B., Peek, D., Chen, P. M., and Flinn, J. 2008. Parallelizing security checks on commodity hardware. SIGPLAN Not. 43, 3, 308-318.

Oplinger, J. AND LAM, M. 2002. Enhancing software reliability with speculative threads. In Proceedings of the International Conference on Architectural Support for Programming Languages and Operating Systems.

Patil, H. AND Fischer, C. 1997. Low-cost, concurrent checking of pointer and array accesses in C programs. In Software Practice and Experience.

Patil, H. AND Fischer, C. N. 1995. Efficient run-time monitoring using shadow processing. In Proceedings of the 2nd International Workshop on Automated and Algorithmic Debugging.

Plakal, M. AND Fischer, C. N. 2000. Concurrent garbage collection using program slices on multithreaded processors. In Proceedings of the 2000 International Symposium on Memory Management.

Qin, F., Lu, S., ANd ZHou, Y. 2005. SafeMem: Exploiting ECC-memory for detecting memory leaks and memory corruption during production runs. In Proceedings of the International Symposium on High Performance Computer Architecture.

Rational Software. 2006. Purify. http://www.rational.com/products/purify_unix/index.jsp.

RENAU, J. ET AL. 2004. SESC. http://sesc.sourceforge.net.

Roth, A. AND Sohi, G. 2001. Speculative data-driven multithreading. In Proceedings of the 7th International Symposium on High Performance Computer Architecture.

Savage, S., Burrows, M., Nelson, G., Sobalvarro, P., and Anderson, T. 1997. Eraser: A dynamic data race detector for multithreaded programs. ACM Trans. Comput. Syst. 15, 4, 391-411.

SEWARD, J. 2004. Valgrind, an open-source memory debugger for x86-GNU/Linux. http://valgrind.kde.org.

SEwARD, J. AND Nethercote, N. 2005. Using Valgrind to detect undefined value errors with bit-precision. In Proceedings of the Annual Conference on USENIX Annual Technical Conference. USENIX Association, Berkeley, CA, 2-2.

Shetty, R., Kharbutli, M., Solihin, Y., and Prvulovic, M. 2006. HeapMon: A helper-thread approach to programmable, automatic, and low-overhead memory bug detection. IBM J. Res. Dev. 50, 2/3, 261.

Shetty, R., Kharbutli, M., Solinin, Y., And Prvulovic, M. 2004. HeapMon: A low overhead, automatic, and programmable memory bug detector. In Proceedings of IBM Watson Conference on Interaction between Architecture, Circuits, and Compilers $(P=a c 2)$.

Stern, U. And Dill, D. L. 1995. Automatic verification of the SCI cache coherence protocol. In Proceedings of the Conference on Correct Hardware Design and Verification Methods.

TeAm, T. G. 2008. GNU Compiler Collection. http://gcc.gnu.org.

Tiwari, D., LeE, S., Tuck, J., AND Solihin, Y. 2010. Mmt: Exploiting fine-grained parallelism in dynamic memory management. In Proceedings of the 24th International Parallel and Distributed Processing Symposium.

VAlgrind Developers. 2005. The Valgrind Quick Start Guide. http://valgrind.org/docs/manual/quickstart.html.

Wang, P. H., Collins, J. D., Wang, H., Kim, D., Greene, B., Chan, K.-M., Yunus, A. B., Sych, T., Moore, S. F., AND SHEN, J. P. 2004. Helper threads via virtual multithreading. IEEE Micro 24, 74-82.

Xekalakis, P., Ioannou, N., And Cintra, M. 2009. Combining thread level speculation helper threads and runahead execution. In Proceedings of the 23rd International Conference on Supercomputing.

Zhou, P., Liu, W., Fei, L., Lu, S., Qin, F., Zhou, Y., Midkiff, S., And Torellas, J. 2004a. AccMon: Automatically detecting memory-related bugs via program counter-based invariants. In Proceedings of the $37 \mathrm{th}$ International Symposium on MicroArchitecture.

Zhou, P., Qin, F., Liu, W., Zhou, Y., And ToRellas, J. 2004b. iWatcher: Efficient architectural support for software debugging. In Proceedings of the 31st International Symposium on Computer Architecture.

Received August 2012; revised May 2013; accepted July 2013 